\title{
FORMATION MODELS OF GALAXIES
}

\author{
S.D.M. WHITE \\ Max-Planck-Insitut für Astrophysik \\ Karl-Schwarzschild-Strasse 1, D-8046 Garching bei München, \\ Germany \\ (swhite@MPA-Garching.mpg.de)
}

Note by the editors:

Unfortunately Dr. White was not able to finish a written version of his presentation at the Symposium in time for inclusion in these proceedings. He refers the interested readers to his lecture notes at the August 1993 Les Houches summer school (White, 1995).

\section{References}

White, S.D.M., 1995, "Formation and Evolution of Galaxies", in 1993 Les Houches Lectures on Large-scale Structure, ed. R. Schaeffer, Elsevier, Netherlands. 